\title{
Acute Appendicitis Presented as a Cause of Sigmoid Colon Obstruction: A Case Report
}

\author{
Adel R. Al-Masry ${ }^{1}$, Ifrat Bakirov ${ }^{2}$, Aly Saber ${ }^{3}$, Junaid Hassan ${ }^{4}$ \\ ${ }^{1}$ Department of General Surgery, Al-Mahalla Al-Koubra General Hospital, Al-Mahalla Al- Koubra, Egypt \\ ${ }^{2}$ Department of General Surgery, Huraymala General Hospital, Al- Ryadh, KSA \\ ${ }^{3}$ Department of General Surgery, Port-Fouad General Hospital, Port-Fouad, Egypt \\ ${ }^{4}$ Department of General Surgery, Mayo Hospital, KEMU, Lahore, Pakistan \\ Email address: \\ adelrm2003@yahoo.com (A. R. Almasry), Alysaber54@gmail.com (A. Saber)
}

\section{To cite this article:}

Adel R. Al Masry, Ifrat Bakirov, Aly Saber, Junaid Hassan. Acute Appendicitis Presented as a Cause of Sigmoid Colon Obstruction: A Case Report. Journal of Surgery. Special Issue: Gastrointestinal Surgery: Recent Trends. Vol. 4, No. 2-1, 2016, pp. 1-3.

doi: $10.11648 /$ j.js.s.2016040201.11

\begin{abstract}
Background: Acute appendicitis is a common surgical problem. However, the diagnosis is often overlooked when it presents as a bowel obstruction. Case presentation: In this report we present a case of elderly patient presented with bowel obstruction and radiological signs of sigmoid colon volvulus. Although there were no accurate manifested signs of acute appendicitis it was the real cause of acute large bowl obstruction. The patient was successfully treated with a laparotomy, adhesiolysis and appendicectomy and went on to make a good recovery. Conclusion: Acute appendicitis should be considered in the differential diagnosis of patients with large bowel obstruction.
\end{abstract}

Keywords: Obstruction, Sigmoid Colon, Acute Appendicitis

\section{Introduction}

Acute appendicitis is a very common disease with low morbidity and mortality rates in most countries[1].Intestinal obstruction is a common surgical emergency caused by varied conditions. Appendix as a cause of intestinal obstruction is uncommon and not usually suspected. Although it was described as early as1901, very few reports are available which do a comprehensive review[2]. Pre-operatively, it is very difficult to diagnose this condition. The diagnosis is always made at the time of laparotomy. The treatment varies from appendicectomy to intestinal resection or even right hemicolectomy[3]. We present here a case of acute appendicitis presented clinically as bowel obstruction, radiologicaly as sigmoid volvulus.

\section{Case Presentation}

A 51 year Sudanese male farmer was admitted to emergency department with history of acute severe abdominal pain, located mainly peri-umbilical for one week and constipation four days ago with nausea and vomiting. General examination revealed temperature $36.8^{\circ} \mathrm{C}$, pulse $84 \mathrm{~b} / \mathrm{m}$ and blood pressure 114/67. The patient was known to be diabetic uncontrolled. No past history of previous operations. Local abdominal examination showed mild distension, tenderness all over the abdomen, with rebound tenderness. The abdomen was soft and lax. Bowel sound sluggish. Blood picture shows normal leucocytic count $(9.8 \times 103 / \mu \mathrm{L})$ with relative neutrophilia $75.8 \%, \quad \mathrm{Hb} 14.8 \mathrm{~g} / \mathrm{dL}$ other blood chemistry values were normal, random blood sugar was 7.9 mmol(N,3.9-6.1 mmol), blood urea nitrogen was high11.6mmol/L normal creatinine level, total bilirubin was 28umol/L direct 7.6umol/L, serum amylase normal.

A radiographic film of the abdomen demonstrated a huge air-filled distended bowel in the shape of an inverted " $U$ ", with the convexity of the "U" facing the right upper abdominal quadrant. Contrast enema showed dilatation in the sigmoid colon with an area of complete obstruction gave the appearance of "bird-beak" sign; the right colon is distended with signs of fecal stasis fig (1).

U/S abdomen showing distended bowels. CT abdomen without contrast gave the appearance of dilatation of large bowel loops, concluded? Sigmoid volvulus figure (2). 


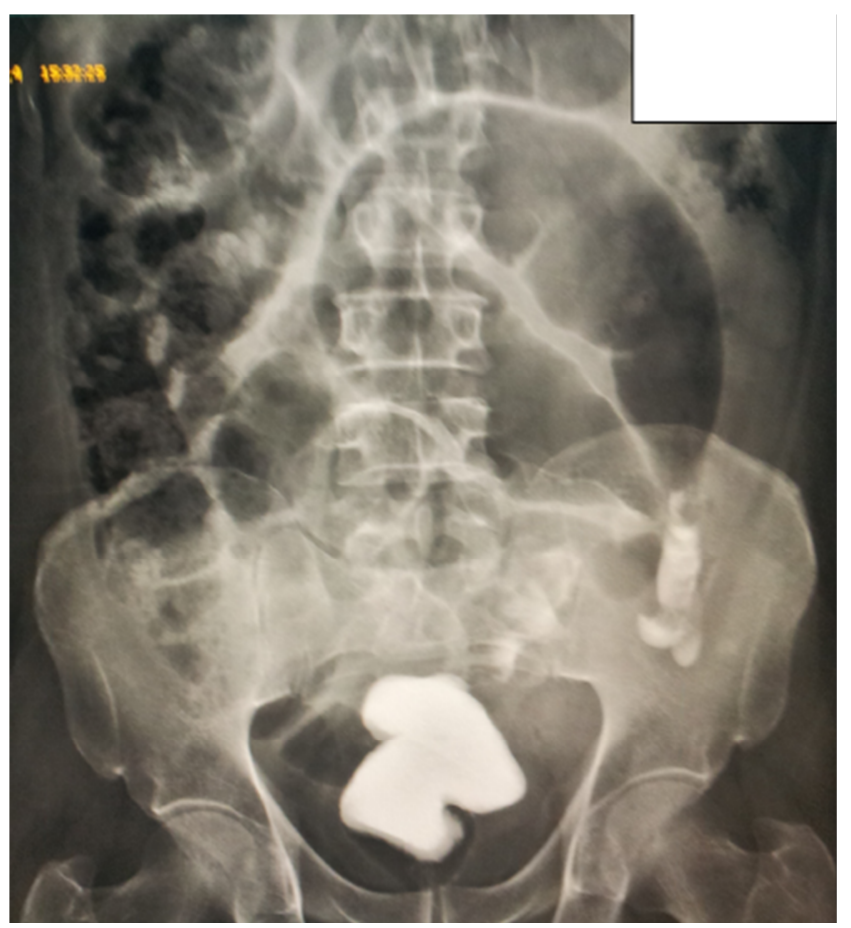

Figure (1). A preoperative plain X-ray film of the abdomen demonstrated a huge air-filled distended bowel in the shape of an inverted " $U$,"with its convexity facing the right upper abdominal quadrant.

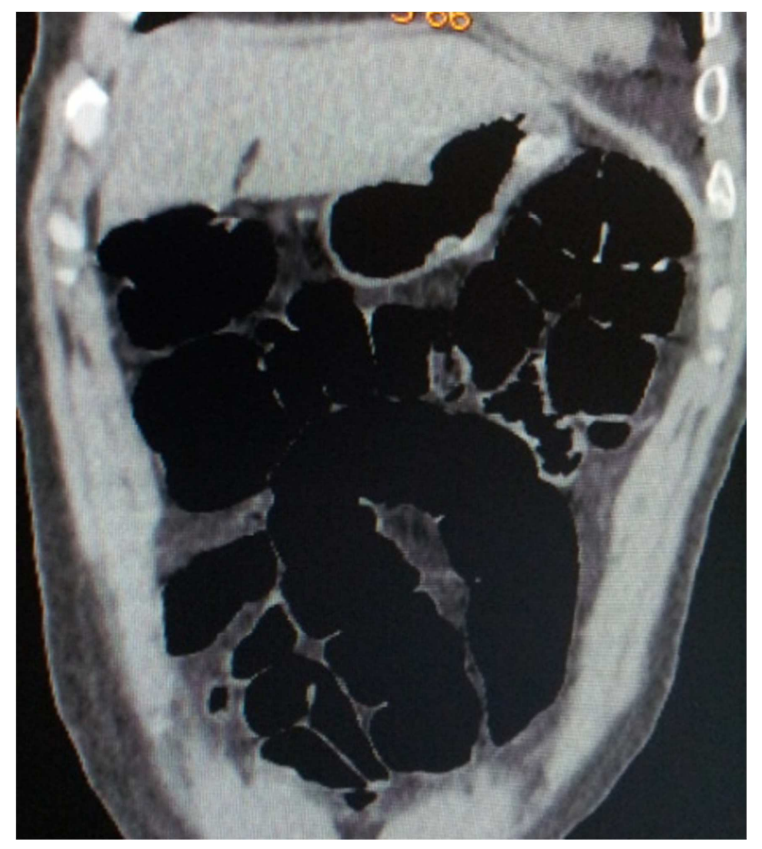

Figure (2). A preoperative CT scan film of abdomen without contrast gave the appearance of dilatation of large bowel loops, concluded? Sigmoid volvulus.

The patient was putted under conservative measures for 24 hours. Meanwhile patient was kept nil per oral with naso-gastric aspiration and insertion of rectal tube. When the free end of the rectal tube was putted under water surface, it reveals air bubbling, denoting that the obstruction was not complete one. The patient was started on prophylactic intravenous antibiotics and analgesics, without improvement of the obstructive manifestations with appearance of toxic manifestations. Lab revealed rising of leucocytic count with more neutrophil, total bilirubin (direct), and serum amylase. Exploratory laparotomy was done through midline incision reveals distended sigmoid colon but not found to be hugely distended and not twisted counter-clockwise around its root-mesentry. The appendix had pelvic position, and was adherent to the two limbs of pelvic colon near its mesentry-root by adhesions producing the picture of inverted "U" shape of sigmoid volvulus that presented in plain X-ray, contrast enema and CT abdomen. Pelvis had too many adhesions that released and revealed gangrenous perforated appendicitis in pelvic position. Peritoneal swap was taken, later on proved to be E.coli organism. Retrograde appendicectomy was done. Abdominal cavity washed with 3L saline. Drain closure and dressing. Pathological examination revealed acute suppurative gangrenous perforated appendicitis. Postoperative period was un-eventful and patient was discharged on $7^{\text {th }}$ day, to be followed up in out-patient department.

\section{Discussion}

The presentation of acute appendicitis in the elderly can be atypical, resulting in a delayed diagnosis with potential for increased morbidity and mortality. Presentation with mechanical bowel obstruction may pose further challenges[4,5].

Neglected un-treated acute appendicitis may passed un-noticed and presented later by different picture e.g. adhesive intestinal obstruction, small or large intestine, as well as generalized peritonitis, appendix abscess, liver abscess. The clinical features of bowel obstruction may dominates the clinical picture and mask acute appendicitis[4].

Large bowel obstructions are far less common than small bowel obstructions, accounting for only $20 \%$ of all bowel obstructions[6]. The underlying aetiology of large bowel obstructions is age-dependant, but in adult-hood, the most common cause is colonic cancer (50-60\%), typically in the sigmoid colon[6-9].

The second most common cause in adults is acute diverticulitis (involving the sigmoid colon). Together, obstructing tumours and acute diverticulitis account for $90 \%$ of all causes of large bowel obstruction. While adhesions are the leading cause of small bowel obstruction, for practical purposes, they do not tend to cause large bowel obstruction[9].

Overall causes of large bowel obstruction include malignancy such as colo-rectal carcinoma and pelvic tumors, colonic diverticulitis, volvulus such as caecal volvulus and sigmoid volvulus[9]. Other causes include ischaemic stricture, faecal impaction/faecoloma and hernias but as an uncommon cause[10].

Our patient was immunocompromised with diabetes mellitus which predisposes to rapid inflammatory process, and during the initial events of appendicular inflammation, it would get adherent to the surrounding structures, producing various pathology; gangrene, perforation of the appendix and adhesive sigmoid colon obstruction as the appendix had pelvic position. 
The present case is considered rare, as neglected un-treated acute appendicitis cause mainly adhesion and obstruction in small bowel not large bowel and it doesn't mentioned before in causes of large bowel obstruction. In this case the history, clinical examination and investigations were correlated with sigmoid colon volvulus.

The appendix in the present case had pelvic position, and was adherent to the two limbs of pelvic colon near its mesentery root, the adhesions producing the picture of inverted "U" shape of sigmoid volvulus that presented in plain $\mathrm{X}$-ray, contrast enema and $\mathrm{CT}$ abdomen. At laparotomy the pelvic colon was found not so hugely distended and not twisted counter-clockwise around its root-mesentery, but the two limbs were adherent near the root mesentery, together with the inflamed appendix underneath. However in sigmoid volvulus, a long, redundant sigmoid colon is the major cause of sigmoid volvulus, this redundant, enlarged bowel causes the approximation of two limbs of sigmoid colon and predisposes the limbs to twist around the mesenteric axis[11].

The diagnosis of sigmoid volvulus is made by physical examination and radiographic studies. Abdominal radiographs demonstrate a markedly distended sigmoid colon with a convex superior margin projecting into the right upper quadrant of the abdomen. This section of sigmoid colon is often devoid of haustral markings. A "coffee bean" or "omega loop" sign has been described on abdominal radiograph, these terms refer to the two large compartments of distended sigmoid colon with central double walls of colon and a single outer wall, which assume the shape of a coffee bean or omega loop[12]. Computed tomography scan has been used to rule out other etiologies of obstruction and colonic ischemia in patients with sigmoid volvulus. On radiography, a "bird's beak" sign can be demonstrated at the torsion point of the sigmoid[13]. However, in our case abdominal radiography showed inverted "U" shape directed towards right upper quadrant, but not the shape of a coffee bean nor omega loop appearance, also contrast enema showed a "bird's beak" sign, provided with clinical manifestation of colonic obstruction so, it resemble a challenge in its diagnosis.

\section{Conclusion}

Acute appendicitis should be considered in the differential diagnosis of patients with large bowel obstruction. To the best of the authors' knowledge, this finding has not been described previously.

\section{References}

[1] Blomqvist PG, Andersson RE, Granath F, Lambe MP, Ekbom AR: Mortality after appendectomy in Sweden, 1987-1996. Annals of surgery 2001, 233(4):455-460.

[2] Hotchkiss Lucius W: Acute intestinal obstruction following appendicitis. Areport of three cases successfully operated upon. Ann Surg1901, 34:660-677.

[3] Bhandari L and Mohandas PG: Appendicitis as a cause of intestinal strangulation: a case report and review. World Journal of Emergency Surgery 2009, 4:34 doi: 10. 1186/ 1749 - $7922-$ 4-34.

[4] Bose S, Talwar B: Appendicitis causing acute intestinal obstruction with strangulation. The Australian and New Zealand Journal of Surgery1973, 43(1):56-57.

[5] Acute appendicitis presenting as small bowel obstruction: two case reports Sanjay Harrison*1, Kamal Mahawar1, Dougal Brown 2, Leslie Boobis 1 and Peter Small 1 Cases Journal 2009, 2:9106doi:10.1186/1757-1626-2-9106.

[6] Khurana B, Ledbetter S, Mctavish J et-al. Bowel obstruction revealed by multidetector CT. AJR Am J Roentgenol. 2002; 178(5):1139-44.

[7] Tjandra J J, Clunie G J, Kaye A H. Text book of Surgery. Wiley-Blackwell. (2006) ISBN: 1405126272.

[8] Choi J S, Lim J S, Kim Het-al. Colonic pseudo obstruction: CT findings. AJRAm J Roentgenol.2008; 190(6):1521-6.

[9] Brant W E, Helms C A. Fundamental sof Diagnostic Radiology. Lippincott Williams \&Wilkins. (2007)ISBN: 0781761352.

[10] Aguirre D A, Santosa A C, Casola Get-al. Abdominal wall hernias: imaging features, complications, and diagnostic pitfalls at multi-detector row CT. Radiographics. 2005; 25(6): 1501-20.

[11] Imbembo A L, Zucker K A. Volvulus of the colon. In: Sabiston D C (ed). Text book of Surgery, The Biological Basis of Modern Surgical Practice, 14th edition. Philadelphia, W.B. Saunders Company, 199, pp.940-944.

[12] Salati U, Mcneill G, Torreggiani W C. The coffee bean sign in sigmoid volvulus. Radiology. 2011; 258 (2): 651 - 2. doi: $10.1148 /$ radiol.101882.

[13] Mallory Williams, MD, Christopher P. Steffes, MD: Casereport: Sigmoid Volvulus in a 46-Year-Old Man www. turner-white. com Hospital Physician January 2006. 